\title{
Performance evaluation of the V2I fair access with a finite retry limit
}

\author{
Qiong $\mathrm{Wu}^{1,3}$, Hao Zhang ${ }^{1}$, Zhengquan $\mathrm{Li}^{2,3^{*}}$, Yang Liu ${ }^{1}$ and Cui Zhang ${ }^{4}$
}

\begin{abstract}
This paper evaluates the performance of the vehicle-to-infrastructure (V2I) fair access with a finite retry limit through constructing an analytical model. Firstly, the process of the distributed coordination function (DCF) mechanism with a finite retry limit is described through developing a 2-D Markov chain; based on the Markov chain, the transmission probability with the access parameters including the minimum window and the retry limit is derived. Then, considering the V2I unfair access problem, the relationship between the velocity and the transmission probability of a vehicle is derived. Based on the proposed model, given a finite retry limit, the minimum window of a vehicle can be determined according to its velocity. In the simulation, the proposed model is justified to be effective and the V2I fair access performance with a finite retry limit is evaluated through comparison.
\end{abstract}

Keywords: Performance evaluation, Fair access, V2l, Retry limit

\section{Introduction}

Vehicular networks can provide safety and entertainment applications through the vehicle-to-infrastructure (V2I) communication. In a vehicular network, multiple vehicles with different velocities usually need to access a roadside unit (RSU) using a single channel, which would lead to an unfair access problem, i.e., the vehicles which move with different velocities would spend different time to move in the communication range of the RSU and thus spend different time to transmit packets to the RSU. To solve the V2I unfair access problem, it is useful to design an access scheme to achieve V2I fair access.

To solve the V2I unfair access problem, Karamad and Ashtiani proposed a fair access scheme base on the distributed coordination function (DCF) mechanism [1]. The fair access scheme allows a vehicle to change its minimum window adaptively to realize fair access. The performance of the fair access scheme is analyzed through constructing a model [1]. This protocol is proposed based on the DCF mechanism. The DCF mechanism specifies that for each packet, the number of retransmission should not be larger

\footnotetext{
* Correspondence: Izq722@sina.com

${ }^{2}$ State Key Laboratory of Networking and Switching Technology, Beijing

University of Posts and Telecommunications, Beijing, China

${ }^{3}$ National Mobile Communications Research Laboratory, Southeast University,

Nanjing, Jiangsu, China

Full list of author information is available at the end of the article
}

than a finite number, i.e., retry limit [2]. A packet would be dropped after the number of retransmission reaches the finite retry limit. However, this work assumes that the retry limit is infinite and each vehicle always has packets prepared for transmitting. In the real world, a vehicle would not always have packets prepared for transmitting. In our prior work, we have constructed models to analyze the V2I fair access performance when each vehicle does not always have packets prepared for transmitting [3, 4]. However, our prior work does not consider a finite retry limit. Without a retry limit, vehicles may retransmit a packet consecutively when the channel contention level is high. The retry limit is an important factor of the DCF mechanism. It specifies a maximum time for retransmissions and thus affects the access performance of the DCF mechanism. Extensive work has focused on the access of vehicular networks [5-27]. However, these work does not consider the V2I unfair access problem. It is necessary to construct a model to evaluate the V2I fair access performance with a finite retry limit. This is our motivation.

In this paper, we evaluate the V2I fair access performance with a finite retry limit through constructing an analytical model. Firstly, a 2-D Markov chain is developed to model the process of the DCF mechanism with a finite retry limit; based on the Markov 
chain, the transmission probability with the access parameters including the minimum window and the retry limit is derived. Then, considering the V2I unfair access problem, the relationship between the velocity and the transmission probability of a vehicle is derived. Based on the proposed model, given a finite retry limit, the minimum window of a vehicle can be determined according to its velocity. In the simulation, the proposed model is justified to be effective and the V2I fair access performance with a finite retry limit is evaluated through comparison.

This paper is organized as follows. Section 2 describes the network scenario and reviews related work. Section 3 proposes a Markov chain to model the process of the DCF mechanism with a finite retry limit and derives the transmission probability with the access parameters including the minimum window and the retry limit. Section 4 considers the V2I unfair access problem and derives the relationship between the velocity of a vehicle and the transmission probability to achieve the V2I fair access. Section 5 evaluates the V2I fair access performance with retry limit through simulation. Section 6 concludes this paper.

\section{Related work}

We introduce the V2I communication scenario in a vehicular network firstly and then review the related work in this section.

\subsection{Network scenario}

In a vehicular network, multiple vehicles with different velocities usually need to access a RSU through a channel, which would lead to an unfair access problem. To evaluate the V2I fair access performance, a V2I communication scenario is considered as follows. Multiple vehicles with different velocities move on a road and each vehicle does not change its moving direction. A RSU is deployed on the road side. The vehicles are assumed to arrive in the communication range of the RSU in groups. Each group arrives in the communication range of the RSU according to a Poisson process with an arrive rate $\lambda$. The number of vehicles in each group is distributed uniformly in $[0, b]$. The velocities of the vehicles in each group are identical and remain a constant when the vehicles are within the communication range of the RSU. Moreover, the vehicles need to communicate with the RSU once they are within the communication range of the RSU. Figure 1 shows an example with three groups.

All vehicles in the network communicate with the RSU employing the DCF mechanism. Each vehicle accesses a channel with a same retry limit and a same minimum window. In this condition, each vehicle transmits packets with a same transmission probability. However, when vehicles which move with different velocities pass through the communication range of the RSU, they would spend different time to communicate with the RSU. This would result in that the vehicles which move with higher velocity would have less opportunity to transmit packets compared with the vehicles moving with lower velocity. This problem is the unfair access problem, which can affect the access performance largely.

\subsection{Related work}

Extensive work has focused on the access of vehicular networks [5-27]. In [5], Bianchi constructed a Markov chain to evaluate the DCF performance. In $[6,7]$, Duffy et al. constructed a Markov chain to evaluate the DCF performance when each node does not always have packet to transmit. In [8], Sun and Dai constructed a framework to observe the effect of the retry limit on the

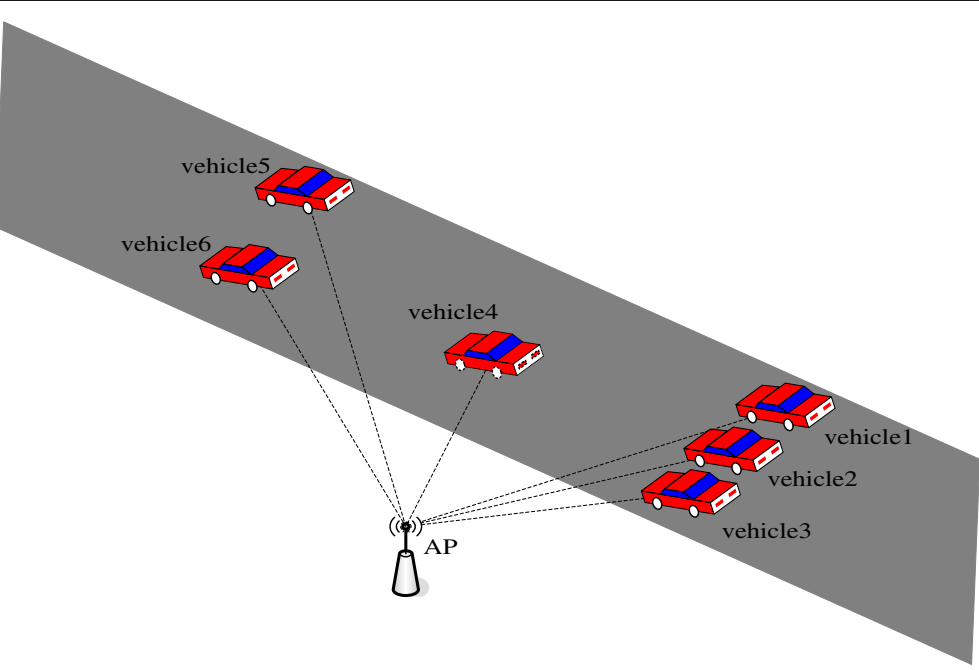

Fig. 1 Network scenario 
performance optimization of the DCF mechanism. In [9], Banchs et al. constructed a model to evaluate the 802.11aa performance with retry limit. In [10], Gupta and Rai proposed a model to evaluate the performance of the DCF mechanism with a retry limit. The proposed model considers both the saturation traffic and the nonsaturation traffic. In [11], Babich et al. proposed an algorithm to jointly manage the voice and video access categories through justifying the retry limit adaptively and evaluated the performance of the proposed algorithm. In [12], Rashwand et al. constructed a model for the access mechanism of the 802.15.6 in a wireless body network. The constructed model takes into account an errorprone channel, a retry limit and a packet queue. In [13], Zheng et al. construct a model to evaluate the DCF performance considering the group-synchronization. In [14], Liao et al. proposed an access scheme to improve the access performance and constructed a model to investigate the benefit of the proposed access scheme. In [15], Swain et al. evaluated the DCF performance including the throughput, delay, and power consumption considering different traffic conditions and a power save mode. In [16], Madhavi and Rao proposed a new packet access process based on the DCF mechanism to decrease the number of collision and increase the throughput. Moreover, they constructed a framework to evaluate the performance. In [17], $\mathrm{Li}$ et al. proposed a model to predict a vehicular network performance considering a Rayleigh channel. In $[18,19], \mathrm{Wu}$ and Zheng proposed models to evaluate the performance of the IEEE 802.11p EDCA mechanism. In [20, 21], Wu and Zheng proposed models to evaluate the performance of the ADHOC MAC protocol. In [22], Chen et al. proposed a cross-tier handover algorithm based on a preset threshold policy to increase the received signal to interference plus noise ratio (SINR) at the macrocell base station/femtocell access point (MBS/FAP) in two-tier Femtocell networks. In [23], Xiong et al. proposed a broad beamforming approach (BBA) to increase the signal-tonoise ratio (SNR). In [24], Lu et al. adjusted power to improve the handover performance in high-speed railway communications systems. In [25], Xiong et al. proposed a mobile service amount based link scheduling (MSA-LS) for high-mobility vehicular networks to overcome the inefficiency of current instantaneous rate based link scheduling (IIRLS) method. In [26], Li et al. evaluated the transmission performance in the high-speed railways (HSRs) and derived an optimal power allocation strategy for HSRs. In [27], Xiong et al. proposed a novel fairness power allocation scheme to increase the high mobile service amount with fairness between the water-filling and proportional power allocation. However, these work mentioned above does not consider the unfair access problem in vehicular networks, which motivated us to conduct this work.

\section{Modeling for the DCF mechanism}

In this section, we first describe the DCF mechanism briefly and then construct a Markov chain to model the access process of the DCF mechanism with a finite retry limit.

\subsection{DCF mechanism}

The DCF mechanism uses a backoff scheme to access a channel. Each node which has packets to transmit senses the channel firstly. If the node senses that the channel stays idle continuously within a duration distributed interframe space (DIFS), it would transmit the packet. Otherwise, the node would start a backoff process. In this case, it will start up a counter. Initially, the value of the counter is selected from $\left[0, W_{\min }\right]$ randomly; here, $W_{\min }$ denotes the minimum window. Then, after each time slot, the value of the counter will be deduced by 1 . After the value of the counter is deduced to 0 , the node would transmit the packet. If an acknowledgement (ACK) packet is not received in a short inter-frame space (SIFS) duration, a collision occurred and the node would retransmit the packet. The value of $W_{\min }$ will be doubled firstly at the initial of each retransmission; then, the node chooses another number from $\left[0, W_{\min }\right]$ randomly as the value of the counter and starts a new backoff process. After $W_{\min }$ reaches $W_{\max }$, where $W_{\max }$ is the maximum window, it will not be doubled and keep the value $W_{\max }$. If the number of retransmissions is larger than the retry limit, the node would drop the packet and reset the value of $W_{\text {min }}$ to the initial value, and then, the node would start a new backoff process. If the node has no packet to be transmitted and the counter is decremented to 0 , the node would keep the counter to be 0 until it has a packet to transmit.

\subsection{Markov chain for the backoff process}

To derive the performance of the DCF mechanism with a finite retry limit, we establish a Markov chain to model the backoff process. The Markov chain considers a finite retry limit and the condition that each node does not always have packet to transmit. Based on the Markov chain, the transmission probability with access parameters including the minimum window and the retry limit is derived. The notations in the Markov chain are introduced in Table 1.

Like most related work [3-17], we assume that the collision probability is equal for different transmission and the channel is ideal; a collision occurred only when two nodes within a same communication range transmit packets at the same time.

We establish a Markov chain to describe the backoff process, which is shown in Fig. 2. The backoff process of the DCF mechanism can be described in Section 3.1.

The one-step transition probabilities can be obtained according to the backoff process. According to 
Table 1 Notations used in the Markov model

\begin{tabular}{|c|c|}
\hline Notation & Definition \\
\hline$i$ & The number of the retransmission \\
\hline k & The value of a counter \\
\hline$b(i, k)$ & $\begin{array}{l}\text { Stationary probability of states with a packet waiting to } \\
\text { transmit when the number of the retransmission is } i \text { and the } \\
\text { value of the counter is } k\end{array}$ \\
\hline$b(i, k)_{e}$ & $\begin{array}{l}\text { Stationary probability of states without packets waiting to } \\
\text { transmit when the number of the retransmission is } i \text { and the } \\
\text { value of the counter is } k\end{array}$ \\
\hline$W_{m}^{i}$ & Window size when the number of the retransmission is $i$ \\
\hline$m$ & $\begin{array}{l}\text { The maximum number of the retransmission beyond which } \\
\text { the window would not be doubled }\end{array}$ \\
\hline$m+f$ & Retry limit \\
\hline 9 & Probability that a node has a packet prepared to transmit \\
\hline$p$ & Collision probability \\
\hline$P_{\text {idle }}$ & Probability that the channel is sensed idle \\
\hline$\tau$ & Transmission probability \\
\hline
\end{tabular}

the one-step transition probabilities, the transmission probability can be expressed as follows:

$$
\tau=b(0,0)_{\mathrm{e}}\left(\frac{W_{0}}{(1-p)\left[1-(1-q)^{W_{0}}\right]}-P_{\text {idle }}\right) \frac{q^{2}\left(1-p^{m+f+1}\right)}{1-q}
$$

According to the one-step transition probability, $b(0,0)_{\mathrm{e}}$ can be expressed as follows:

$$
\begin{aligned}
& \frac{1}{b(0,0)_{e}}=(1-q)+\frac{p q^{2}}{2(1-p)(1-q)} \cdot\left[\frac{W_{0}}{1-(1-q)^{W_{0}}}-(1-p) P_{\text {idle }}\right] \\
& \left\{W_{0} \times \frac{\left[1-(2 p)^{m}\right](1-p)}{1-2 p}+1+W_{0}(2 p)^{m}\left(1-p^{f}\right)-p^{m+f}\right\}+\frac{q\left(W_{0}+1\right)}{2(1-q)} . \\
& {\left[\frac{q^{2} W_{0}}{1-(1-q)^{W_{0}}}+\left(1-P_{\text {idle }}\right)(1-q)-q P_{\text {idle }}(1-p)\right]+\frac{q^{2} W_{0}\left[W_{0}+1\right]}{2\left[1-(1-q)^{W_{0}}\right]}}
\end{aligned}
$$

\section{Performance analysis for the V2I fair access}

In this section, we analyze the fair access performance with a finite retry limit.

The network scenario is shown in Fig. 1. The V2I fair access is considered, and the relationship between the velocity and the transmission probability is derived. Combining the obtained transmission probability in Section 3, we derive the relationship between the minimum window and the velocity to achieve the V2I fair access.

The relationship between the velocity and the transmission probability is derived firstly. According to [1], the transmission rate of vehicle $i$ can be expressed by

$$
M_{i}=S \times \frac{R_{b}}{N_{\mathrm{v}}} \times \frac{\tau_{i}}{\sum_{k=1}^{N_{\mathrm{v}}} \tau_{k}},
$$

where $S$ denotes the normalized throughput of the networks, $R_{b}$ denotes the rate of the channel, $N_{\mathrm{v}}$ denotes the number of vehicles, and $\tau_{i}$ denotes the transmission probability of vehicle $i$.

To access a channel fairly, during the time each vehicle passes through the communication range of the RSU, each vehicle should transmit the same number of packets:

$$
M_{i} T_{i}=C, \quad T_{i}=\frac{R}{v_{i}},
$$

where $T_{i}$ denotes the time that vehicle $i$ passes through the communication range of the RSU, $R$ denotes the communication range of the RSU, $v_{i}$ denotes the velocity of vehicle $i$, and $C$ denotes a constant.

We substitute Eq. (3) to Eq. (4) and have the following:

$$
S \times \frac{R_{b}}{N_{v}} \times \frac{\tau_{i}}{\sum_{k=1}^{N_{v}} \tau_{k}} \times T_{i}=C .
$$

In Eq. (5), let $C^{\prime}=S \times \frac{R_{b}}{N_{\mathrm{v}}} \times \frac{1}{\sum_{k=1}^{N_{\mathrm{v}}} \tau_{k}} . C^{\prime}$ is independent of $i$ and thus is a constant. Eq. (5) can be rewritten as

$$
\tau_{i} T_{i}=K_{\text {index }}, K_{\text {index }}={ }^{C} / C^{\prime}, T_{i}=\frac{R}{v_{i}},
$$

where $K_{\text {index }}$ is the fairness index, which is a constant. In another word, to access a channel fairly, each vehicle in the network should have the same fairness index $K_{\text {index }}$. Here, we obtain the relationship between the velocity and the transmission probability to achieve V2I fair access.

Then, we approximate the relationship between the minimum window and transmission probability. In the considered network scenario, the vehicles arrived at the network in group, and the vehicles in the same group have the same access parameters. The parameters are described in Table 2.

In Eq. (1) and Eq. (2), the transmission probability depends on the minimum window $W$. In the DCF mechanism, the minimum window is much larger than 1, i.e., $W_{i} \gg 1$ [2]. Therefore, the following approximation can be obtained:

$$
\left(1-\tau_{i}\right)^{n_{i}-1} \approx\left(1-\tau_{i}\right)^{n_{i}}
$$

The above approximate is more accurate for the larger value of $n_{i}$. Based on Eq. (7), the collision probability for group $i$ can be derived:

$$
p_{i}=1-\left(1-\tau_{i}\right)^{n_{i}-1} \prod_{k=1, k \neq i}^{B}\left(1-\tau_{k}\right)^{n_{k}} \approx 1-\prod_{k=1}^{B}\left(1-\tau_{k}\right)^{n_{k}},
$$




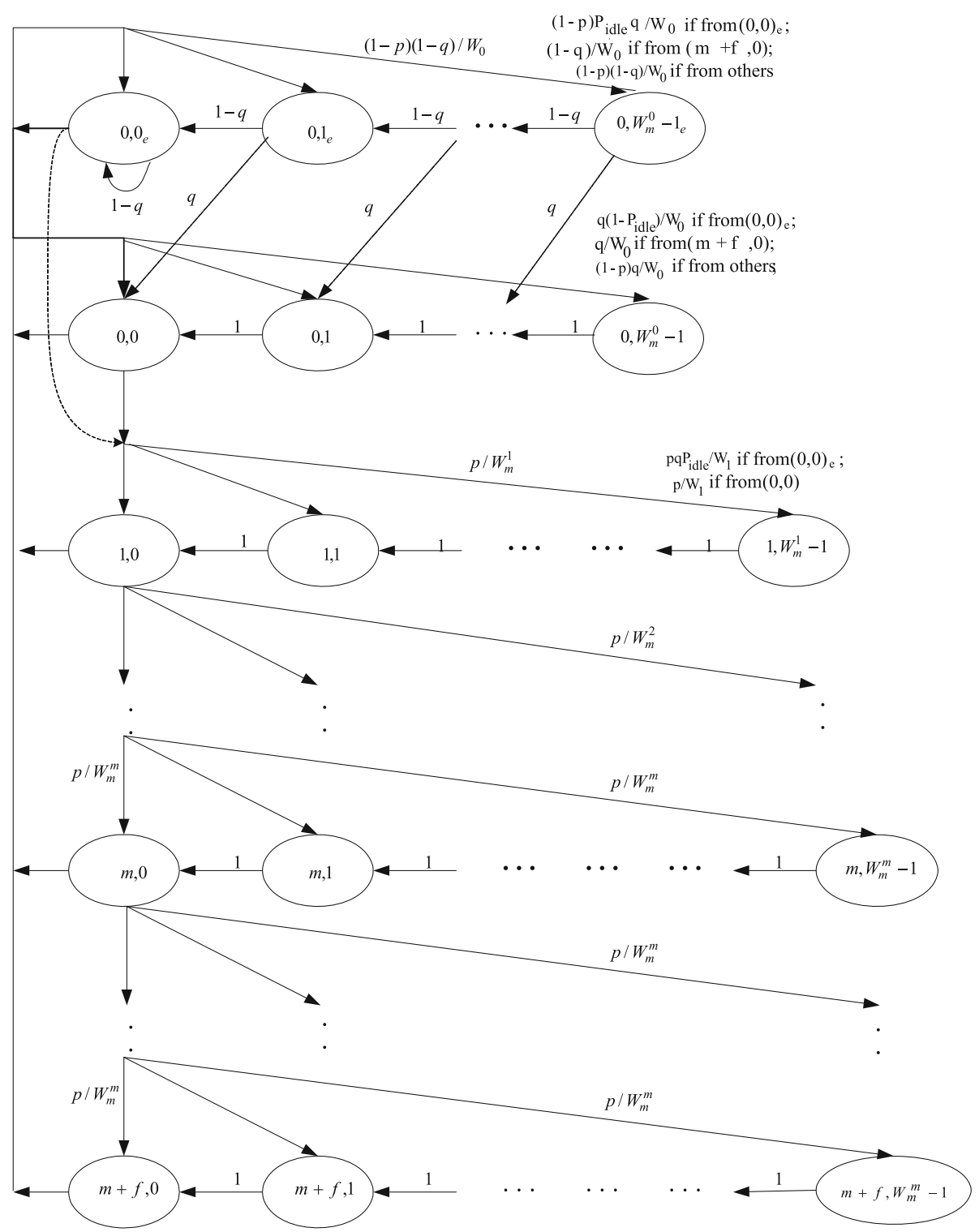

Fig. 2 Markov chain for the backoff process

where the number of groups within the communication range of the RSU is $B$. Therefore, the collision probability for group $i$, i.e., $p_{i}$, can be approximated to a constant $p$.

Using the similar approximation method in [3], the approximation results of the transmission

Table 2 Parameters of a vehicle in group $i$

\begin{tabular}{ll}
\hline$T_{i}$ & $\begin{array}{l}\text { The time that a vehicle passes through the } \\
\text { communication range of the RSU }\end{array}$ \\
$T_{i}$ & The transmission probability \\
$V_{i}$ & The velocity \\
$n_{i}$ & The number of vehicles \\
$W_{i}$ & The minimum window \\
$P_{i d l e}^{i}$ & The probability that the channel is sensed idle
\end{tabular}

probability can be obtained, which can be expressed as follows.

$$
\tau_{i} \approx \frac{1}{W_{i} \times\left\{\frac{1-p}{2\left(1-p^{m+f_{m}+1}\right)}+\frac{p(1-p)\left[1-(2 p)^{m}\right]}{(1-2 p)\left(1-p^{m+f_{m}+1}\right)}+\frac{p(2 p)^{m}\left(1-p^{f}\right)}{2\left(1-p^{m+f_{m}+1}\right)}\right\}} .
$$

According to Eq. (8), $p$ can be approximated to a constant. According to Eq. (9), the relationship between the minimum window and the transmission probability for group $i$ can be obtained:

$$
\tau_{i}=\frac{1}{W_{i} f(p)}=\frac{K_{1}}{W_{i}},
$$


Table 3 Parameters used in the simulation

\begin{tabular}{ll}
\hline$T_{p}(\mu \mathrm{s})$ & 8184 \\
$T_{s}(\mu \mathrm{s})$ & 8972 \\
$T_{c}(\mu \mathrm{s})$ & 8713 \\
$\mathrm{ACK}(\mu \mathrm{s})$ & 240 \\
DIFS $(\mu \mathrm{s})$ & 128 \\
$\mathrm{SIFS}(\mu \mathrm{s})$ & 28 \\
Slot time $(\mu \mathrm{s})$ & 50 \\
Vehicles' velocity(m/s) & Uniform in $[5,45]$ \\
$D(\mathrm{~m})$ & 500 \\
Mean group size & 2 \\
$m$ & 3 \\
$f$ & 8 \\
Average minimum window & 64 \\
$T(s)$ & 50 \\
\hline
\end{tabular}

$$
\begin{aligned}
f(p)= & \frac{1-p}{2\left(1-p^{m+f_{m}+1}\right)}+\frac{p(1-p)\left[1-(2 p)^{m}\right]}{(1-2 p)\left(1-p^{m+f_{m}+1}\right)} \\
& +\frac{p(2 p)^{m}\left(1-p^{f_{m}}\right)}{2\left(1-p^{m+f_{m}+1}\right)}, K_{1} \\
= & \frac{1}{f(p)} .
\end{aligned}
$$

Since $p$ is approximated to a constant, both $f(p)$ and $K_{1}$ are constant. Moreover, according to Eq. (10), we can find that the transmission probability for a group is approximated to be not dependent on $q$. Here, we obtain the approximation relationship between the minimum window and the transmission probability.
Finally, the relationship between the minimum window and the velocity can be obtained. According to Eq. (6) and Eq. (10), we can obtain the following equations:

$$
\tau_{i} T_{i}=K_{\text {index }} \text { and } \tau_{i}=\frac{K_{1}}{W_{i}} \text {, where } \quad T_{i}=\frac{R}{v_{i}} .
$$

Combining the above equations, the relationship between the minimum window and the velocity for group $i$ can be obtained:

$$
v_{i} \times W_{i}=K_{2}, \text { where } K_{2}=\frac{K_{1} \times R}{K_{\text {index }}} .
$$

From Eq. (12), we can find that $K_{2}$ is a constant. Moreover, we can find that the minimum window is in inverse proportion to the velocity for a group.

Averaging both side of Eq. (12), the relationship between the average minimum window and the average velocity can be obtained:

$$
\bar{W} \times \bar{v}=K_{2},
$$

where the average minimum window is $\bar{W}$ and the average velocity is $\bar{v}$.

The average minimum window can be known according to the DCF mechanism, and in the real world, the average velocity can be calculated according to the velocities of vehicles recorded by RSU. From Eq. (13), we can calculate $K_{2}$ according to $\bar{W}$ and $\bar{v}$. Moreover, for a vehicle, the velocity is known. Therefore, the minimum window can be calculated according to Eq. (12).

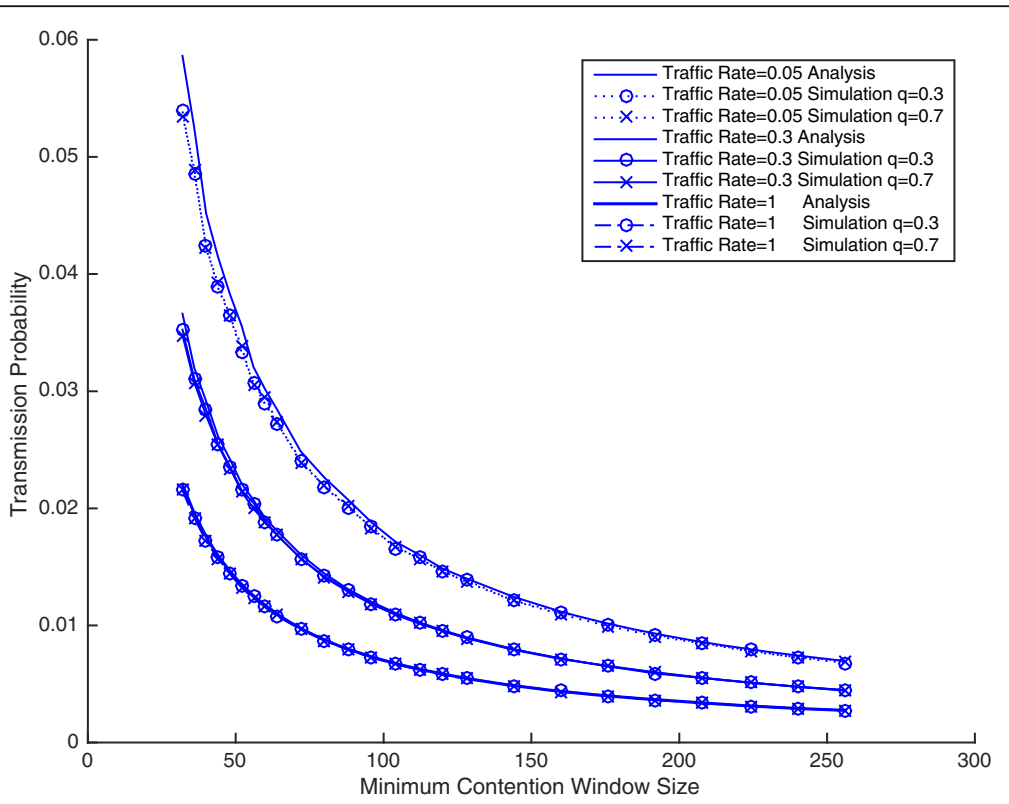

Fig. 3 Minimum window vs transmission probability 


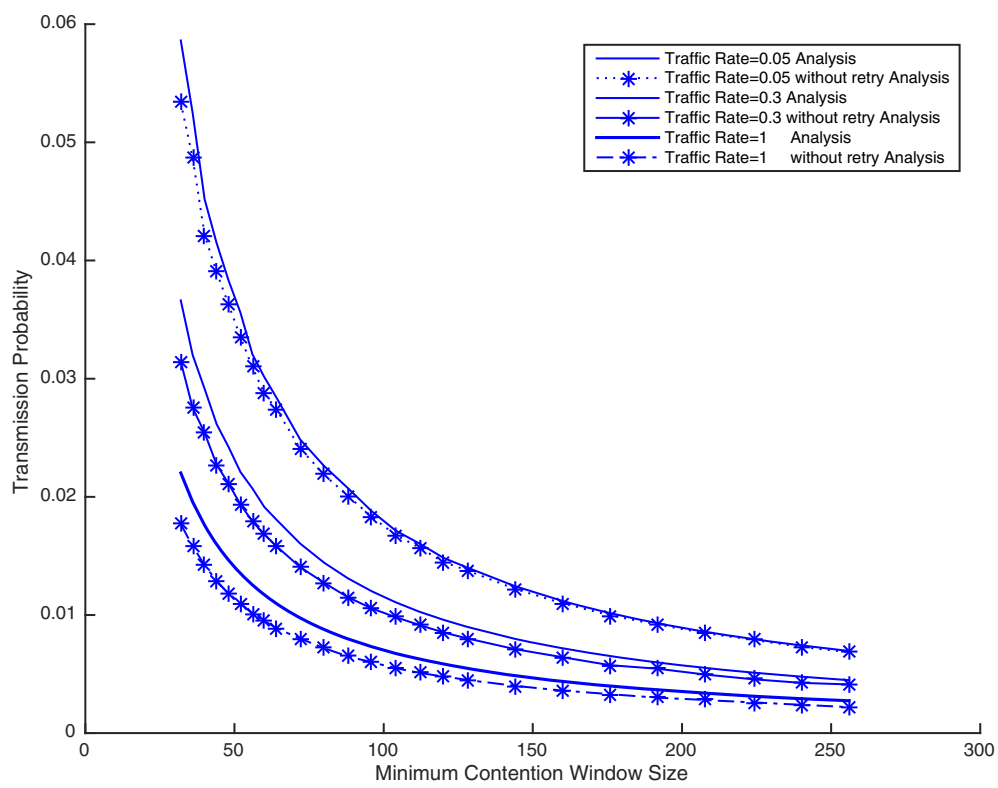

Fig. 4 Transmission probability with a retry limit vs without a retry limit

\section{Simulation results}

The analytical results derived in Section 4 are verified through simulation results in this section. Moreover, we compare the derived analytical results with the analytical results derived in [3], i.e., the analytical results without considering the retry limit, to evaluate the performance of the V2I fair access with a retry limit. The simulation scenario is shown in Fig. 1; the vehicles arrive in the communication range of the RSU in groups with an arrive rate $\lambda$. Once a vehicle arrives at the communication range of the
RSU, it has a packet to transmit with probability $q$. Let $T$ be the simulation time, $T_{s}$ be the duration time of a successful transmission, $T_{p}$ be the duration time of a packet, and $T_{\mathrm{c}}$ be the duration of a collision $T_{\mathrm{c}}$. The parameters of the DCF mechanism are set according to the 802.11 standard [2]. Table 3 shows the value of the parameters.

The relationship between the minimum window and transmission probability under different $\lambda$ and $q$ is shown in Fig. 3. In this simulation experiment, the velocity is $25 \mathrm{~m} / \mathrm{s}$. The simulation results are almost the same with

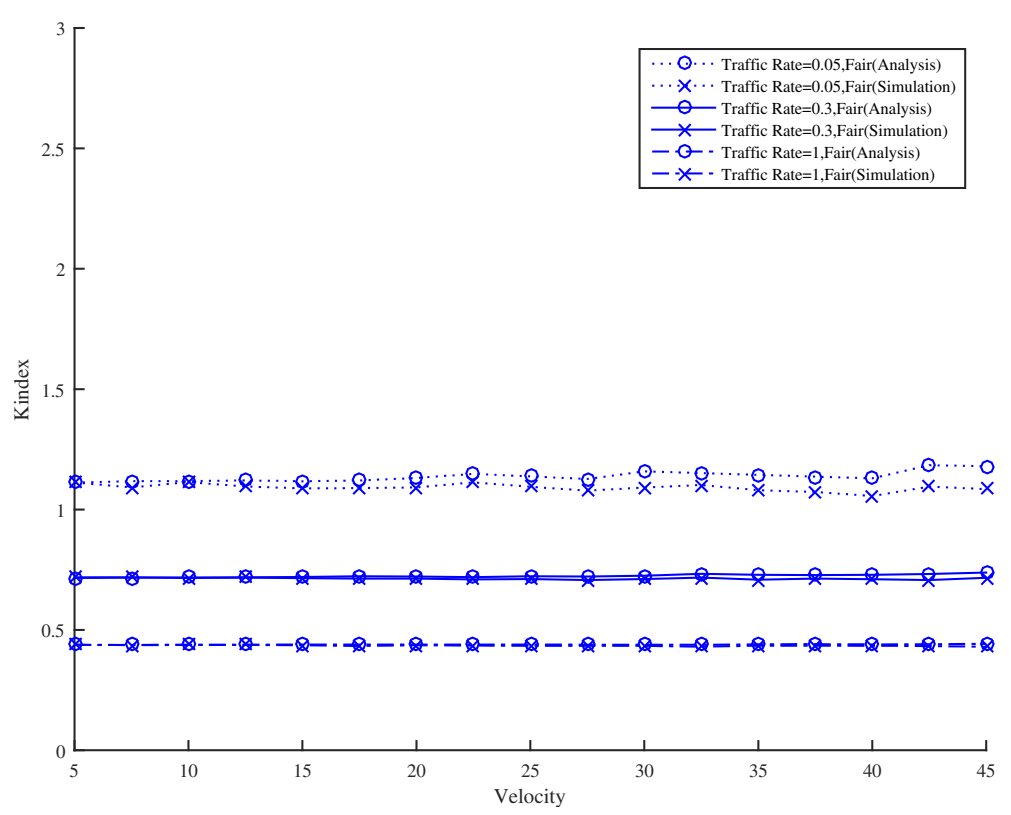

Fig. 5 Velocity vs $K_{\text {index }}$ 


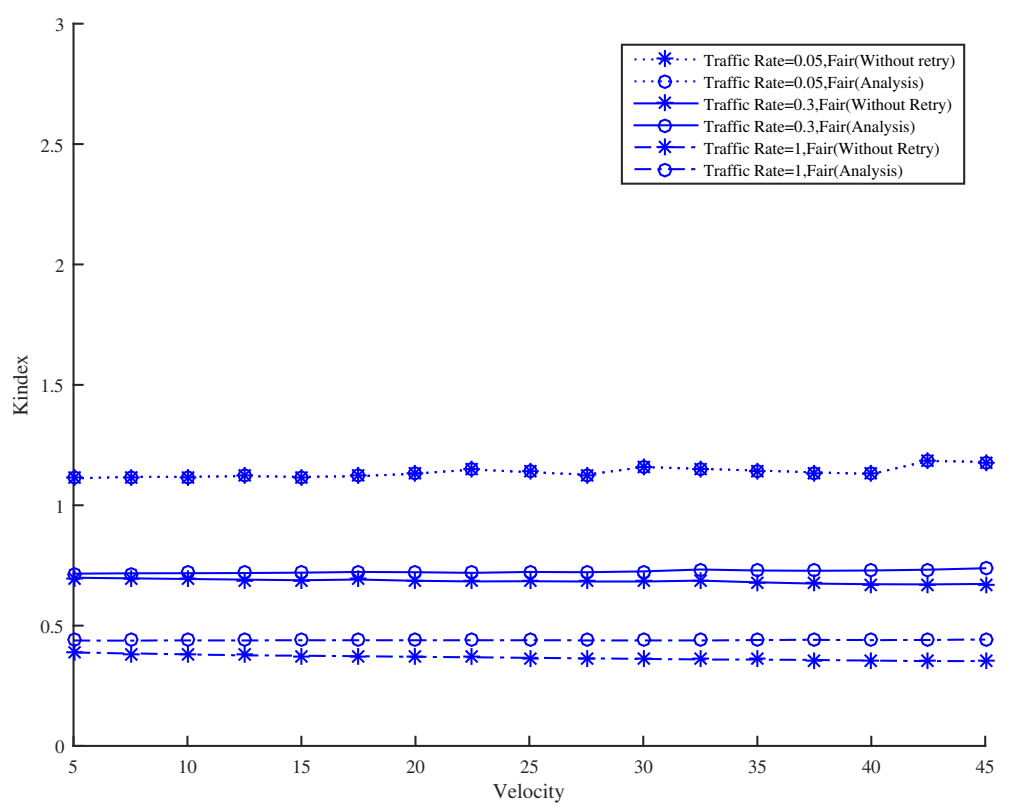

Fig. $6 K_{\text {index }}$ with a retry limit vs without a retry limit

the analytical results, which means the performance analysis is accurate. In Fig. 3, the transmission probability is in inverse proportion to the minimum window and the transmission probability is not dependent on $q$ for a particular value of $\lambda$, which justified the results of the Eq. (11).

Figure 4 compares the derived analytical transmission probability with the analytical transmission probability which does not consider the retry limit. It is seen that the transmission probability considering a retry limit is larger when the minimum window is small. The reason is that the backoff counter would be deduced to 0 frequently and a packet is often prepared to retransmit when the minimum window is small. If a retry limit is considered, the packet would be dropped when the number of the retransmission is larger than the retry limit, and hence, the packet would not be transmitted frequently with a large backoff counter. In this case, the transmission probability considering a retry limit is larger when the minimum window is small.

The relationship between the velocity and $K_{\text {index }}$ is shown in Fig. 5. The traffic rate equals 0.05 , and the minimum commend window size is 64 in this experiment. We can see that the simulation results are almost the same with the analytical results, i.e., the performance analysis is accurate. In Fig. 5, the value of $K_{\text {index }}$ almost does not change with velocity increasing, which verifies Eq. (6).

Figure 6 compares the derived analytical fairness index $K_{\text {index }}$ with the analytical $K_{\text {index }}$ which does not consider a retry limit. The value of $K_{\text {index }}$ considering a retry limit is smaller than that value without considering a retry limit. This is because that the fairness index is in direct proportion to the transmission probability according to Eq. (6).

\section{Conclusions}

This paper evaluates the performance of the V2I fair access with a finite retry limit through constructing an analytical model. Firstly, a 2-D Markov chain is developed to model the process of the DCF mechanism with a finite retry limit; the transmission probability with access parameters including the minimum window and the retry limit is derived based on the Markov chain. Then, considering the V2I unfair access problem, the relationship between the velocity and the transmission probability of a vehicle is derived. Based on the proposed model, given a finite retry limit, the minimum window of a vehicle can be determined according to its velocity. In the simulation, the proposed model is justified to be effective and the V2I fair access performance with a finite retry limit is evaluated through comparison.

\section{Funding}

This work was supported by the National Natural Science Foundation of China under Grant Nos. 61701197, 61571108, 61701284, and 61761030; the Open Research Fund of National Mobile Communications Research Laboratory,

Southeast University, under Grant No. 2018D15; the China Postdoctoral Science Foundation Funded Project under Grant Nos. 2017M622233 and 2017M622103: the Open Foundation of State Key Laboratory of Networking and Switching Technology, Beijing University of Posts and Telecommunications, under Grant No. SKLNST-2016-2-14; the Open Foundation of Key Laboratory of Wireless Communication, Nanjing University of Posts and Telecommunication, Jiangsu Province, under Grant No. 2017WICOM01; and the Fundamental Research

Funds for the Central Universities under Grant Nos. JUSRP11742 and JUSRP11738.

\section{Authors' contributions}

QW and ZL conceived and designed the experiments. $\mathrm{HZ}$ and $\mathrm{QW}$ performed the experiments. $\mathrm{HZ}$, QW, and $\mathrm{CZ}$ analyzed the data. $\mathrm{QW}, \mathrm{HZ}$, and $\mathrm{YL}$ wrote the paper. All authors read and approved the final manuscript.

Competing interests

The authors declare that they have no competing interests. 


\section{Publisher's Note}

Springer Nature remains neutral with regard to jurisdictional claims in published maps and institutional affiliations.

\section{Author details \\ ${ }^{1}$ Key Laboratory of Advanced Process Control for Light Industry, Jiangnan University, Wuxi, Jiangsu 214122, China. ${ }^{2}$ State Key Laboratory of Networking and Switching Technology, Beijing University of Posts and Telecommunications, Beijing, China. ${ }^{3}$ National Mobile Communications Research Laboratory, Southeast University, Nanjing, Jiangsu, China. ${ }^{4}$ Huawei Technologies Co., Ltd, Beijing, China.}

Received: 18 November 2017 Accepted: 2 January 2018

Published online: 22 January 2018

\section{References}

1. E Karamad, F Ashtiani, A modified 802.11-based MAC scheme to assure fair access for vehicle-to-vehicle communications. Comput. Commun. 31(12), 2898-2906 (2008)

2. IEEE 802.11. Part 11: Wireless LAN Medium Access Control (MAC) and Physical Layer (PHY) Specifications, 1997

3. Q Wu, J Zheng, Performance modeling and analysis of IEEE 802.11 DCF based fair channel access for vehicle-to-roadside communication in a nonsaturated state. Wirel. Netw 21(1), 1-11 (2015)

4. Q Wu, J Zheng, Performance Modeling of IEEE 802.11 DCF Based Fair Channel Access for Vehicular-to-Roadside Communication in a Non-saturated State (Proc. of IEEE International Conference on Communications(ICC), Sydney, 2014), pp. 2575-2580

5. G Bianchi, Performance analysis of 802.11 distributed coordination function. IEEE Journal on Selected Areas in Communications 18(3), 535-547 (2000)

6. K Duffy, D Malone, D Leith, Modeling the 802.11 distributed coordination function in non-saturated conditions. IEEE Communication Letters 9(8), 717-715 (2005)

7. D Malone, K Duffy, D Leith, Modeling the 802.11 distributed coordination function in non-saturated heterogeneous conditions. IEEE/ACM Transaction on Networking 15(1), 159-172 (2007)

8. X Sun, L Dai, Performance optimization of CSMA networks with a finite retry limit. IEEE Trans. Wirel. Commun. 15(9), 5947-5962 (2016)

9. A Banchs, A Qlica, L Eznarriaga, D Kowalsk, P Sereano, Performance analysis and algorithm selection for reliable multicast in IEEE 802.11 aa wireless LAN. IEEE Trans. Veh. Technol. 63(8), 3875-3891 (2014)

10. N Gupta, C Rai, Non-saturation Throughput Analysis of IEEE 802.11DCF Considering Short Retry Limit for Single Hop ad hoc Networks (Proc. of 2013 second international conference on future generation communication technology(FGCT), London, 2013), pp. 10-15

11. F Babich, M Comisso, R Corrado, F Merazka, Joint voice/video retry limit adaptation for on-demand streaming over WiFi networks. IEEE Commun. Lett. 20(9), 1852-1855 (2016)

12. S Rashwand, J Misic, V Misic, Analysis of CSMA/CA mechanism of IEEE 802 15.6 under non-saturation regime. IEEE Transactions on Parallel and Distributed Systems 27(5), 1279-1288 (2016)

13. L Zheng, M Ni, L Cai, J Pan, C Ghosh, K Doppler, Performance analysis of group-synchronized DCF for dense IEEE 802.11 networks. IEEE Trans. Wirel. Commun. 13(11), 6180-6192 (2014)

14. R Liao, B Bellalta, M Oliver, Modelling and enhancing full-duplex MAC for single-hop 802.11 wireless networks. IEEE Wireless Communications Letter 4(4), 349-352 (2015)

15. P Swain, S Chakraboty, S Nandi, P Bhaduri, Performance modeling and analysis of IEEE 802.11 IBSS PSM in different traffic conditions. IEEE Trans. Mob. Comput. 14(8), 1536-1233 (2015)

16. T Madhavi, G Rao, Modelling collision alleviating DCF protocol with finite retry limits. Electron. Lett. 51(2), 185-187 (2015)

17. B Li, S Chen, G Sutton, Y Shi, R Liu, MAC performance analysis for drive-thru Internet networks with Rayleigh capture. IEEE ACCESS 5, 10649-10661 (2017)

18. J Zheng, Q Wu, Performance modeling and analysis of the IEEE 802. $11 \mathrm{p}$ EDCA mechanism for VANET. IEEE Trans. Veh. Technol. 65(4), 2673-2687 (2016)

19. Q Wu, J Zheng, Performance Modeling of the IEEE 802.11p EDCA Mechanism for VANET (proc. of IEEE Globe Communication Conference(GLOBECOM), Austin, 2014), pp. 57-63

20. $\mathrm{Q}$ Wu, J Zheng, Performance modeling and analysis of the ADHOC MAC protocol for vehicular networks. Wirel. Netw 22(3), 799-812 (2016)
21. Q Wu, J Zheng, Performance Modeling and Analysis of the ADHOC MAC Protocol for VANETs (proc. of IEEE International Conference on Communications (ICC), London, 2015), pp. 3646-3652

22. G Chen, J Zheng, L Shen, A preset threshold based cross-tier handover algorithm for uplink co-channel interference mitigation in two-tier Femtocell networks. Wirel. Netw 22(6), 1819-1835 (2016)

23. K Xiong, B Wang, C Jiang, L Liu, A broad beamforming approach for highmobility communications. IEEE Trans. Veh. Technol. 66(11), 10546-10550 (2017)

24. $Y$ Lu, K Xiong, P Fan, Z Zhong, B Ai, The effect of power adjustment on handover in high-speed railway communication networks. IEEE ACCESS 5 26237-26250 (2017)

25. K Xiong, Y Zhang, P Fan, $H$ Yang, X Zhou, Mobile service amount based link scheduling for high-mobility cooperative vehicular networks. IEEE Trans. Veh. Technol. 66(10), 9521-9533 (2017)

26. T Li, K Xiong, P Fan, K Letaief, Service-oriented power allocation for highspeed railway wireless communications. IEEE ACCESS 5, 8343-8356 (2017)

27. K Xiong, $\mathrm{P}$ Fan, $\mathrm{Y}$ Zhang, $\mathrm{K}$ Letaief, Towards $5 \mathrm{G}$ high mobility: $\mathrm{A}$ fairness-adjustable time-domain power allocation approach. IEEE ACCESS 5, 11817-11831 (2017)

\section{Submit your manuscript to a SpringerOpen ${ }^{\circ}$ journal and benefit from:}

- Convenient online submission

- Rigorous peer review

Open access: articles freely available online

- High visibility within the field

Retaining the copyright to your article 